Nina Berend

\title{
Russlanddeutsche Aussiedler in Deutschland: Ein Überblick
}

\section{Einleitung}

Russlanddeutsche Aussiedler sind vor allem durch ihren spezifischen Migrationshintergrund auffällig. Sie sind gemäß ihres Einwanderungsstatus als Deutsche bzw. Deutschstämmige weder mit nichtrussischsprachigen Ausländern, Gastarbeitern oder Asylsuchenden/Asylberechtigten noch mit „Kontingentflüchtlingen“, ebenfalls russisch-sprachigen Zuwanderern aus der ehemaligen Sowjetunion, zu vergleichen. Vom Einwanderungshintergrund kommen die Russlanddeutschenden den sog. Vertriebenen und Flüchtlingen am nächsten, die als Deutsche aus den ehemaligen Siedlungsgebieten in Osteuropa während und nach dem Zweiten Weltkrieg nach Deutschland umgesiedelt sind (Bausinger 1956). Eine andere Vergleichsgruppe, die in jüngster Zeit auf ähnlicher Basis wie Russlanddeutsche nach Deutschland einwanderte, sind Aussiedler aus den osteuropäischen Ländern. Deutschstämmige Aussiedler betragen nach aktuellen Angaben 78 Prozent aller Zugewanderten in Deutschland. ${ }^{1}$ In Zahlen ausgedrückt stellt sich die Aussiedler-Zuwanderung wie folgt dar: ca. 2,2 Millionen Aussiedler sind aus den Staaten der ehemaligen Sowjetunion (Russland, Kasachstan, Kirgistan, Ukraine, Tadschikistan, Usbekistan, Moldawien, Estland, Lettland, Litauen, Weißrussland, Georgien, Armenien, Aserbaidschan, Turkmenistan) zugewandert. Weitere große Auswanderungsländer sind Polen (1,5 Mio.) und Rumänien (0,5 Mio.). Außerdem hat eine zahlenmäßig geringere, aber doch bedeutende Zuwanderung von Aussiedlern aus Ungarn, Tschechien, der Slowakei und dem ehemaligen Jugoslawien stattgefunden. Aussiedler aus der ehemaligen Sowjetunion sind eine vergleichsweise neue Zuwanderungspopulation: 70 Prozent wanderten in den letzten 20 Jahren ein und über drei Viertel gehört der Einwanderungsgeneration an.

Trotz des spezifischen Hintergrunds der Zuwanderung als Heimkehrer werden die russlanddeutschen Aussiedler in der Migrationsforschung nicht ausdrücklich als Remigranten sondern höchstens als ko-ethnische Migranten untersucht (Currle 2006; Cyrus et al. 2005; bei Cyrus et al. findet sich ein Überblick über die verschiedenen Gruppen und Typen von „returnees“ und „,co-ethnics“). Direkte Vergleiche mit anderen Migrantengruppen in Deutschland, z.B. in Bezug auf die Einschätzung der Integrationserfolge, wurden daher selten durchgeführt. Die Integration dieser Zuwanderergruppe wurde nach spezifischen Kriterien gemessen und in den letzten zwanzig Jahren meistens negativ bewertet, im Unterschied zu früheren Einwanderungsphasen. In einer im Jahr 2009 veröffentlichten Studie des Berlin-Instituts für Bevölkerung und Entwicklung wurden Russlanddeutsche erstmals als Migranten mit anderen, regulären Zuwanderungsgruppen in Deutschland vergleichend untersucht. Die Fragestellung, welche Gruppen von Zugewanderten wo, in welchem Ausmaß und auf welche Weise integriert sind, und welche Gründe für die entsprechenden Konstellationen vorliegen, wurde mit Hilfe des sog. Index zur Messung der Integration (IMI) ermittelt (Woellert et al. 2009). Angesichts der vorherigen Ergebnisse

Quelle und weitere Angaben: Migrationsbericht 2009 des Bundesamts für Migration und Flüchtlinge: www.bamf.de/SharedDocs/Anlagen/DE/Publikationen/Migrationsberichte/migrationsbericht-2009. html). 
der existierenden Forschung über Russlanddeutsche und vor dem Hintergrund der sonst meistens negativen Medienberichte fällt das Ergebnis dieser Studie eher positiv aus. Die Autoren kommen in Bezug auf die Russlanddeutschen zum Schluss, dass es sich bei Aussiedlern um ,eine sehr integrationsfreudige Herkunftsgruppe“ handelt und die Integration der Russlanddeutschen „erstaunlich gut gelungen“ ist. ${ }^{2}$ Dieses Ergebnis basiert auf einigen Faktoren. Neben der Tatsache, dass diese Zuwanderer in allen Regionen Deutschlands und insbesondere in ländlichen Gebieten angesiedelt wurden („Länder-Quote“), hat besonders auch der höhere Stellenwert der Bildung in den Herkunftsländern der Aussiedler eine wichtige Rolle bei der Integration gespielt. Besonders hervorgehoben wird die hohe Zahl von bi-kulturellen Eheschließungen, worin sich auch die gute Integration widerspiegelt:

[...] Aussiedler bleiben nicht unter sich. An der Quote der bi-kulturellen Eheschließungen sieht man, dass sie sich stark mit der einheimischen Bevölkerung vermischen. Von der zweiten Generation der bereits hier geborenen Verheirateten sind 67 Prozent eine Ehe mit Einheimischen eingegangen - das ist sehr viel. Ihr Migrationshintergrund wird so über kurz oder lang verloren gehen. Und Heiraten mit der einheimischen Bevölkerung ist der beste Weg dahin. (Tagesschau 5.5.2014, s. Fußnote 2)

Diese positiven Ergebnisse, die vor allem die zweite Generation der Russlanddeutschen betreffen, können natürlich nicht über die Probleme hinwegtäuschen, mit denen die erste Generation dieser Zuwanderer - trotz ihres ,privilegierten“" Einwanderungs- und Aufenthaltsstatus - zu kämpfen hatte. In der Forschungsliteratur wird direkt darauf hingewiesen, dass die Probleme bei der ko-ethnischen Migration insbesondere die Einwanderungsgeneration betreffen und oft übersehen werden: „The immigration of co-ethnics is often a neglected topic in migration research but is of particular importance for studies of civic participation of first generation immigrants" (Cyrus et al. 2005). Die Untersuchungen im Projekt Migrationslinguistik, das im Anschluss an das Aussiedlerprojekt ${ }^{3}$ im Institut für Deutsche Sprache durchgeführt wird, haben die Absicht, die sprachlich-soziolinguistischen Probleme der koethnischen Migranten der Einwanderungsgeneration aus der ehemaligen Sowjetunion zu beschreiben. Untersucht werden Nachkommen der Angehörigen der deutschsprachigen Minderheit, die in der Sowjetunion als Kinder oder Jugendliche bzw. junge Erwachsene die Deportation aus den angestammten Gebieten im westlichen Russland noch selbst erlebt haben. Für diese Studien wurden Aussiedler aus Russland als Probanden gewonnen, die aus deutschen Sprachinseln in Sibirien stammten, in die ihre Eltern und Großeltern am Anfang des 2. Weltkriegs aus der Ukraine bzw. der WolgaRegion deportiert wurden. Das Ziel ist die Untersuchung der sprachlichen Integration von Russlanddeutschen, die als Erwachsene im Alter von ca. 30 Jahren aus der ehemaligen Sowjetunion nach Deutschland als Angehörige der deutschen Minderheit einwanderten. Um dieses Ziel zu erreichen, wurden u.a. mit mehr als hundert russlanddeutschen Aussiedlern unmittelbar nach ihrer Einwanderung soziolinguistische Tiefeninterviews durchgeführt. ${ }^{4}$ Mit einigen dieser Probanden wurden nach knapp 20 Jahren ihres Aufenthalts in Deutschland die Interviews wiederholt. Außerdem wurden zwischen den beiden Aufnahmezeitpunkten (1992/93 und 2009/10) in Bezug auf einige Probanden teilnehmende Beobachtungen durchgeführt. In einigen Veröffentlichungen wurden bereits Teilergebnisse präsentiert (vgl. Berend 2012), die zeigen, dass die sprachlich-soziolinguistische Integ-

\footnotetext{
2 Siehe www.tagesschau.de/aussiedlerinterview100.html (5.5.2014).

3 Siehe www1.ids-mannheim.de/prag/aussiedler/.

4 Zu den Methoden und Auswertungsergebnissen vgl. Berend (1998).
} 
ration der russlanddeutschen Aussiedler der Einwanderungsgeneration (d.h. koethnischer Migranten) in vielerlei Hinsicht anders verläuft als z.B. bei der Einwanderungsgeneration anderer Zuwanderer.

Worauf ist diese Andersartigkeit zurückzuführen? Die Untersuchungen zeigen, dass bei diesen Zuwanderern in Bezug auf die sprachliche Integration ein unmittelbarer Zusammenhang zwischen der Vormigrationsphase und den ersten Aufenthaltsjahren in Deutschland besteht. Als wichtigster Punkt erweist sich die sprachliche Ausgangsbasis, die Herkunftssprache. Gerade die Konstellation in Bezug auf die Herkunftssprache gestaltet sich bei Aussiedlern grundsätzlich anders. Aussiedler wandern zu diesem Zeitpunkt mehrheitlich mit zwei Herkunftssprachen ein: der Landessprache des Auswanderungslandes (in diesem Fall des Russischen) und der „koethnischen“ Sprache des Ziel- bzw. Einwanderungslandes (in diesem Fall der deutschen Dialekte). Es stellt sich bei ihnen - aus Gründen, die weiter unten noch ausgeführt werden - vor allem die komplexe Frage der Pflege und Förderung der Herkunftssprache Russisch, noch viel mehr und in einem noch größeren Ausmaß als das bei anderen, nicht „koethnischen“ Migranten der Fall ist. Aber auch in Bezug auf die deutsche Herkunftssprache (Herkunftsdialekte) ergeben sich verschiedenartige Fragen des Erwerbs und der Förderung. Der Erhalt von deutschen Dialekten durch die Angehörigen der deutschen Minderheit im Ausland, unter schwierigen Verhältnissen und in fremdsprachiger Umgebung, ist eine Leistung, die nach der Migration in Deutschland häufig keine angemessene Anerkennung fand. Es hat sich gezeigt, dass trotz der häufig vorhandenen Dialektkenntnisse russlanddeutsche Zuwanderer der Einwanderungsgeneration gerade wegen sprachlich-regionaler Andersartigkeit und wegen mangelnder Hochdeutschkenntnisse Schwierigkeiten im Alltag ausgesetzt waren, die nicht nur und nicht ausschließlich auf Sprachschwierigkeiten zurückzuführen sind (im Sinne von Kommunikationsbarrieren wegen fehlender Deutschkenntnisse), sondern auch und gerade mit grundsätzlichen Fragen der Identität sowie Selbst- und Fremdwahrnehmung zusammenhängen. Entscheidend wirkt sich hier aus, dass es sich bei den Herkunftsdialekten der Russlanddeutschen um substandardsprachliche Dialektvarietäten handelt, die in vielerlei Hinsicht in Deutschland nicht den Status als „richtiges Deutsch“ aufweisen (Berend 2012).

\section{Spezifische Problematik der deutschstämmigen Aussiedler}

Im Folgenden werden die spezifischen Probleme der russlanddeutschen Aussiedler skizziert, die Anfang der 1990er Jahre nach Deutschland eingewandert sind. Es zeigt sich, dass die Probleme, mit denen russlanddeutsche Aussiedler der Einwanderungsgeneration und häufig auch der zweiten Generation in Deutschland konfrontiert werden, aufs Engste mit ihrer Situation als Re-Migranten bzw. koethnische Migranten zusammenhängen. Obwohl diese Zuwanderungsgruppe zum Teil bestimmte Vorteile in Anspruch nehmen konnte, können die entstehenden Integrationsprobleme unter Umständen eben auf diese vermeintlichen Vorteile zurückgeführt werden. Die Ergebnisse der Untersuchungen im Rahmen des IDS-Migrationsprojekts zeigen, dass sich die Probleme auf einige Hauptbereiche konzentrieren lassen. Im Folgenden werde ich auf vier Probleme eingehen, die in den Reflexionen der interviewten Probanden am häufigsten thematisiert wurden: 1) ihre Zwischenposition zwischen einheimisch und fremd, 2) ihre gewollte und tatsächliche Identität, 3) die teilweise verwirrenden Folgen in Bezug auf die neue und alte Heimat und 4) die sprachlich-kommunikativen Probleme, die auf die Komplexität der sprachlichsoziolinguistischen Situation, Dialekterhalt und Dialektaufgabe zurückgehen. 


\subsection{Zwischenposition}

Als wichtigstes Problem wird die zugewiesene Zwischenposition wahrgenommen, die Russlanddeutsche nicht nur seitens der deutschen Bevölkerung, sondern auch von der offiziellen Politik her verspüren: Einerseits sind sie deutsche Aussiedler, andererseits Migranten und Menschen mit Migrationshintergrund - ein Widerspruch, der sich bei russlanddeutschen Aussiedlern besonders seit dem Zuwanderungsgesetz 2005 noch verstärkt hat. Im Zusammenhang damit ist hier vor allem die Wahrnehmung von außen als Russen zu nennen. In der Fremdwahrnehmung als Russen spiegelt sich ein Widerspruch zwischen dem Anspruch, als Deutsche eingewandert zu sein, und der Tatsache, als solche aber nicht anerkannt, sondern - wie bei regulären Migranten auch - mit der Nationalität des Herkunftslandes identifiziert zu werden, wider. Die Identifikation mit Russen an sich ist hier weniger ausschlaggebend. Vielmehr kommt es auf die Erfahrung aus Russland an, dort als Deutsche wahrgenommen zu werden. Bei dieser doppelten Fremdheitserfahrung ist auf die Relevanz des Konzepts von Michael Clyne hinzuweisen, der in Migrationssituationen die Berücksichtigung der soziolinguistischen Kontinuität im Herkunfts- und Einwanderungsland eingefordert hat (,sociolinguistic continuity from old to new homeland“, vgl. Clyne 2007). Denn gerade hier zeigt sich der besondere Kontrast zu regulären Migranten (in Deutschland), die in dieser Hinsicht in ihren Heimatländern keine ähnlichen Erfahrungen gemacht haben und bei denen oft mit der Einwanderung nach Deutschland die Migrationsphase bzw. die eigentliche eigene Migrationsgeschichte erst beginnt. Aussiedler haben bereits eine Migrationsgeschichte im Herkunftsland hinter sich, die sich deutlich in Formulierungen wie „Hier die Russen - dort die Deutschen“ widerspiegelt. ${ }^{5}$ Sie wurden im Herkunftsland als Fremde, als Deutsche wahrgenommen - und haben somit bereits Erfahrungen gesammelt, die reguläre Migranten jetzt z.B. in Deutschland machen, wenn sie als „Ausländer“ bzw. „Migranten“ wahrgenommen werden. Eine Gleichstellung mit Russen ist daher aus zweierlei Hinsicht für die Russlanddeutschen problematisch. Erstens wird der Anspruch bzw. das Recht auf Einwanderung dadurch in Frage gestellt, denn sie sind ja als Deutsche eingewandert. Und zweitens wird dadurch die Migrationserfahrung als Deutsche in Russland mit der dort geforderten und teilweise auch realisierten Integration in die russische Gesellschaft als Deutsche einfach außer Acht gelassen bzw. sogar ignoriert. Dabei handelt es sich aber um eine Erfahrung, die Russlanddeutsche geprägt hat und die ihre Besonderheit als koethnische Migranten weitgehend ausmacht und erklärt.

\subsection{Identität}

Ein weiteres Problem der russlanddeutschen Aussiedler ist die Selbstwahrnehmung und der Widerspruch zwischen ,gewollter“ und „tatsächlicher“ Identität. Die durchgeführten Analysen und vorliegenden Daten zeigen, dass jeder Aussiedler der Einwanderungsgeneration sich schon mehr als einmal mit seiner Identität beschäftigt hat. Die Aneignung von russischen kulturellen Eigenheiten infolge der Assimilationsprozesse in Russland ist eine Erscheinung der Integration, die sich in der Regel früher oder später im Einwanderungsland nach der Migration einstellt. In Sprachinseln kann die Integration verzögert werden, wie das bei den Russlanddeutschen bis zum 2. Weltkrieg der Fall war. Durch die Zerstörung der Sprachinseln infolge der Deportation am Anfang des 2. Weltkriegs und Ansiedlung der Russlanddeutschen unter der autochthonen russischen Bevölkerung ist der Integrations- und Assimilationsprozess rasch eingetreten und hat seine Folgen im Laufe der

\footnotetext{
5 Zuletzt haben sich Hermann/Öhlschläger (Hg.) (2013) ausführlich mit der Problematik beschäftigt.
} 
Jahrzehnte der Nachkriegszeit zunehmend gezeigt. Insbesondere die Russlanddeutschen, die in Zerstreuung lebten, d.h. nicht kompakt in deutschen Siedlungen, haben sich nicht nur den russischen kulturellen Eigenheiten angenähert, sondern haben auch - wie aus der jüngsten Geschichte bekannt - insbesondere die russische Sprache angenommen und entsprechend die Kenntnisse der deutschen Dialekte - als wichtigstes Merkmal der deutschen Identität - zum Teil vollständig aufgegeben. Die im Herkunftsland vollzogene teilweise Assimilation spiegelt sich in der Selbstwahrnehmung der Russlanddeutschen wider, die nach der Einwanderung als Deutsche in Deutschland in große persönliche Unsicherheitskonstellationen geraten. Als Ausweg wird oft eine überzogene bzw. anstrengende Anpassung in Deutschland erstrebt, d.h. ein Ausgleich zwischen der gewollten deutschen und tatsächlichen russischen bzw. „fast russischen“ Identität. Das führte in vielen Fällen zu kategorischen Handlungen wie z.B. das „Flüstersyndrom“, wenn Russisch aus dem öffentlichen Gebrauch verbannt wird (Berend 1998) oder wenn die „deutsche Muttersprache“ unter allen Umständen als Familiensprache anstelle des Russischen etabliert werden soll (Diener 2003). Diese von Russlanddeutschen der Einwanderungsgeneration am Anfang ihres Aufenthalts unternommenen „Maßnahmen“ sollten den Sprung von der russischen zur deutschen Identität gewährleisten und zumindest in der Selbstwahrnehmung die Einreiseerlaubnis als „Deutsche“ rechtfertigen.

\section{3 „Heimat“ als Herkunfts- und Zielland}

Wie schwierig sich die Lage der Aussiedler in ihrer spezifischen Migrationssituation infolge der beiden dargestellten Probleme gestaltet, zeigt sich im nächsten Problem. Gerade bei dem Widerspruch zwischen der gewollten und tatsächlichen Identität ist es nicht immer einfach, dem Herkunftsland die Rolle bzw. Funktion als „Heimatland“ abzusprechen. Andererseits ist Deutschland als Zielland ebenfalls Heimatland bzw. neue Heimat der Aussiedler. In zahlreichen Veröffentlichungen und in der Forschungsliteratur wird häufig die Konstellation „Zuhause fremd“ bzw. „Fremde in der Heimat“" (z.B. Ipsen-Peitzmeier/Kaiser (Hg.) 2006) in Bezug auf Aussiedler hervorgehoben. Allerdings wird von vielen interviewten Aussiedlern - trotz aller Missverständnisse und Defizite der Integration - immer wieder als Schlussstrichlinie der Gedanke über Deutschland als Heimat, wenn auch historische Heimat, hervorgehoben. Die Konstellation, die sich dabei einstellt, ist für die Re-Migration der Russlanddeutschen typisch: In gewissem Sinne ist das Herkunftsland Russland bzw. die ehemalige Sowjetunion als Heimat zu sehen, andererseits ist auch das Zielland Deutschland das angestrebte „Heimatland“. ${ }^{6}$ Diese Konstellation ist für „,nicht-koethnische Migranten“ nicht typisch. In der regulären Migration sind das Herkunftsland und Zielland gut voneinander abgetrennt. Migranten können sich in vielerlei Hinsicht in die im Zusammenhang mit dem Herkunftsland entstehenden Nischen zurückziehen. In vielen Befragungen stellte sich heraus, dass z.B. die ethnischen Russen der Einwanderungsgeneration, die mit den Russlanddeutschen in familiären Zusammenhängen nach Deutschland gekommen sind, anders als die ,echten deutschstämmigen“ Familienangehörigen klare Einstellungen zum Herkunftsland Russland und Zielland Deutsch-

6 Hier ist ein Vergleich zu den „Expatriates“ angebracht, denn die Situation ist in vielerlei Hinsicht ähnlich mit der der russlanddeutschen Migranten. Unter der Überschrift „Aus der Fremde in die Fremde“ wird über den „Re-Entry-Schock“ der Expatriates berichtet (www.sueddeutsche.de/karriere/expatriates-ausder-fremde-in-die-fremde-1.1537892): „Wer lange im Ausland arbeitet, muss bei der Rückkehr mit einem zweiten Kulturschock rechnen - denn die Zeiten ändern sich. Und auch im Unternehmen wartet niemand auf den Rückkehrer.“ 
land haben. Russen empfinden Deutschland als Einwanderungsland, in dem sie - mehr oder weniger zufällig - ab einem gewissen Zeitpunkt leben. Sie verbinden damit keinerlei „Heimatgefühle“, sie wollen nicht unbedingt „Deutsche“ werden und haben keine Probleme mit der eindeutigen Identitätszuordnung als Russen, auch wenn es sich ursprünglich um Kasachen, Kirgisen, Tataren usw. aus den Staaten der ehemaligen Sowjetunion handelt.

Anders sieht es bei Familienmitgliedern aus, die deutsche bzw. russlanddeutsche Abstammung haben. Diese fühlen sich dagegen häufig zwischen „,zwei Heimaten“ hin- und hergerissen, d.h. von Unsicherheitsgefühlen überfordert. Durch meine langjährigen Beobachtungen von Russlanddeutschen in Deutschland kann ich die Ergebnisse der soziologischen Forschung bestätigen, die feststellen, dass ,,viele Aussiedler sich selbst als Deutsche definieren, was die Bildung stabiler ethnischer Gemeinschaften verhindert" (Strobl 2006, S. 93). Das führt nicht nur zur eigenen Unsicherheit der Einwanderungsgeneration, sondern ist auch ein Grund dafür, dass auch teilweise die Jugendlichen Unsicherheiten ausgesetzt sind. Einer der Gründe liegt sicherlich darin, dass die Eltern - ausgehend von ihrer Zwischenposition (vgl. 2.1) - keine klare Orientierung haben und oft zwischen allen Stühlen sitzen. Wie Strobl (2006, S. 100) feststellt, führt das in vielen Fällen dazu, dass die Einwanderungsgeneration, d.h. die Generation der Eltern, ,kulturelle Eigenheiten aus der Sowjetunion nicht pflegt, sondern diese möglichst schnell aufgeben möchte und eine schnelle Anpassung an die deutsche Gesellschaft wünscht". Diese Schilderung stimmt auf jeden Fall für die ersten Aufenthaltsjahre der russlanddeutschen Aussiedler der Einwanderungsgeneration ab 1990 bis Ende der 1990er Jahre, wie Forschungen gezeigt haben (vgl. dazu bes. Berend 1998 und Diener 2003). Diener (2003) führt detailliert aus, zu welchen Ergebnissen z.B. die Anstrengungen führen, die deutsche Standardsprache kategorisch als neue Familiensprache einzuführen. Berend (1998) stellt bei dialektsprechenden Russlanddeutschen klare „Verdeutschungstendenzen“ in den ersten Jahren nach der Einwanderung fest.

Wie die Situation bei der Einwanderungsgeneration gegenwärtig aussieht, gut 20 Jahre nach der Einwanderung, ist allerdings fraglich. Es gibt Indizien dafür, dass die Situation sich verändert hat und auch die Elterngeneration sich wieder vermehrt den ,russischen Wurzeln" zuwendet. Für die prägenden ersten Aufenthaltsjahre jedoch war der Widerspruch in Bezug auf „Herkunftsland als Heimat“" und „Zielland als Heimat“" ein relevanter, den Integrationsprozess wesentlich beeinflussender Faktor.

\section{Sprachliche Probleme der Einwanderungsgeneration}

Als letztes soll hier noch das Sprachproblem im eigentlichen Sinne erörtert werden. Russlanddeutsche Aussiedler wandern alle als Bilinguale ein. Doch handelt es sich auf keinen Fall um eine hinsichtlich der Sprachverhältnisse homogene Gruppe. Die Beschaffenheit der deutsch-russischen bzw. russisch-deutschen Zwei- bzw. Mehrsprachigkeit ist bei Sprechern bei der Einwanderung vor allem generationsabhängig. Bei keiner Generation liegt ausgewogene Zweisprachigkeit vor. Meistens ist eine der beiden beherrschten Sprachen die dominante und die andere wird nur domänenspezifisch gebraucht. Es zeigte sich bei der Einwanderung eine spezifische sprachliche Situation, die auf die sprachlichen Repertoires der gesamten Einwanderungspopulation zurückgeht. Versucht man, eine überblicksartige Klassifizierung vorzunehmen, stößt man bald an Grenzen, denn die Sprachverhältnisse sind äußerst heterogen. Die Behauptungen, Russlanddeutsche könnten kein Deutsch 
und würden in der Ingroup-Situation (d.h. in der internen Kommunikation untereinander) nur Russisch oder „sonderbaren Dialekt aus altschwäbischen Resten“ sprechen, wie man sie allgemein oft in den Medien findet, entsprechen nicht der Realität. (Eine typische Darstellung dazu findet sich z.B. in Dworschek 1991.) Bei der Einwanderung kurz nach 1990 lagen große Unterschiede in Bezug auf die Kenntnisse des Russischen und des Deutschen vor. Über fast keine bzw. nur wenige Russischkenntnisse verfügten die älteren Zuwanderer, die aus den Sprachinselgebieten der östlichen Sowjetunion stammten. In der ersten Hälfte der 1990er Jahre machte diese Gruppe einen großen Teil aller aus der Sowjetunion Zugewanderten aus, da in dieser Phase gerade die Zuwanderung aus Sprachinseln ihren Höhenpunkt erreichte. Jugendliche und Erwachsene, die nicht aus Sprachinseln stammten, verfügten in der Regel über sehr gute, oft sogar über muttersprachliche Russischkenntnisse, im Gegensatz zu Jugendlichen und jungen Erwachsenen aus Sprachinseln, die oft noch einen deutschen Akzent aufwiesen (Berend 1977).

Auch in Bezug auf die Kenntnisse des Deutschen bestanden bei der Einwanderung große Unterschiede, die ebenfalls vor allem auf biografische Gegebenheiten der verschiedenen Generationen der Russlanddeutschen zurückgingen. Hier sind grob zwei Gruppen mit unterschiedlichen linguistischen Repertoires zu unterscheiden:

Gruppe 1: Russlanddeutscher Dialekt + etwas Hochdeutsch (dialektbasiert) + Alltagsrussisch (mündlich).

Gruppe 2: kein Dialekt + etwas Hochdeutsch (DaF-Kenntnisse) + Russisch (mündlich und schriftlich).

Natürlich ist dies nur eine grobe Einschätzung der Sprachsituation und der linguistischen Repertoires bei der Einwanderung. Doch schon daran kann man die Problematik erkennen: Die gemeinsame Sprache aller zugewanderten Gruppen ist Russisch. Nicht deutscher Dialekt oder Hochdeutsch, sondern Russisch wurde häufig auch zur Ingroup-Sprache in Familien, und besonders dann, wenn zur Familie auch ethnische Russen gehörten. (Dabei handelte es sich vor allem und am häufigsten um angeheiratete russische Schwiegersöhne.) Russlanddeutsche sprechen somit die Herkunftssprache, die dominante Sprache des Herkunftslandes - so wie alle anderen, regulären Migranten auch. Allerdings, das spezifische Problem, das sich hier in der Wahrnehmung von außen ergibt, lautet: „Deutsche, aber sprechen Russisch." Es geht hier also nicht nur darum, dass Migranten in privater Kommunikation die Herkunftssprache sprechen, was schon an sich von der einheimischen Bevölkerung nicht akzeptiert wird, wie in der Studie zur Einstellung der ,echten“ Deutschen gegenüber fremdsprachigen Akzenten festgestellt wurde (Eichinger et al. 2009). In Bezug auf Russlanddeutsche ist der Gebrauch des Russischen bei den Einheimischen auch deswegen auffällig, weil Aussiedler als Deutschstämmige bzw. „Deutsche“ nach Deutschland einwandern.

Abgesehen davon ist das Russische in Deutschland die Sprache, die nicht besonders beliebt ist: Der russische Akzent ist nach der oben erwähnten Studie der am wenigsten beliebte und nicht gern gehörte unter allen anderen in Deutschland anzutreffenden Akzenten. Dass es sich hier nicht um ein rein linguistisches Problem handelt, sondern dass auf der Grundlage des Akzents auch Identitätszuschreibungen stattfinden, geht aus vielen Äußerungen von Aussiedlern hervor. Wenn Russlanddeutsche in Russland als Deutsche wahrgenommen wurden - häufig auch auf der Grundlage des Akzents, insbesondere bei Angehörigen der älteren Generationen - so geschieht in Deutschland genau das Gegenteil: 
Sie werden aufgrund ihres russischen Akzents und ihrer Sprechweise als Russen wahrgenommen. Durch die Wahrnehmung des russischen Akzents werden die fremdsprachigen Varietäten als slawisches Außendeutsch bezeichnet und die Sprecher entsprechend als Russen, d.h. Nicht-Deutsche, eingeordnet. Treffend hat das eine ältere Aussiedlerin in einer Aussage auf Russisch festgehalten, die hier stellvertretend für viele andere angeführt wird:

Там рот откроешь - ты немеи, здесь рот откроешь - ты уже не немеи!

„Öffnest dort den Mund - bist du Deutscher, öffnest hier den Mund - bist du kein Deutscher mehr!““

\subsection{Spezifische Probleme der russlanddeutschen Dialektsprecher}

Doch nicht nur der russische Akzent ist für manche Integrationsprobleme der russlanddeutschen Aussiedler aus der ehemaligen Sowjetunion verantwortlich. Interessanterweise sind das auch die deutschen Dialekte, die von Aussiedlern im Herkunftsland erhalten wurden und die - anders als in früheren Zeiten - in mancherlei Hinsicht nicht immer auf Anerkennung stoßen. Zum russischen Akzent kommt auch noch ein Dialektakzent hinzu, der für einheimische Ohren ebenfalls oft auffällig ist. Dann ist es ein ,,sonderbarer Dialekt aus altschwäbischen Sprachresten“, der klingt „wie im schwäbischen Dorf im vorigen Jahrhundert". 7 Trotz der Bemühungen, richtiges Hochdeutsch zu sprechen, sind Dialektmerkmale und insbesondere Ausspracheeigenheiten im Hochdeutschen der Aussiedler natürlich nicht zu überhören. In Medien wird das ,etwas abgestandene, in Sprachkursen mühselig vitalisierte Deutsch“ zelebriert und Aussiedler, die sich anstrengen, das ,richtige“ Hochdeutsch zu erwerben, mit Aussagen wie „Mir sagen haite tschuiss“ zitiert (Dworschek 1991). In diesen Wahrnehmungen der einheimischen Bevölkerung - und ihren Widerspiegelungen in den Medien - zeigt sich das eigenartige sprachliche Problem der Russlanddeutschen als koethnischer Migranten besonders deutlich: Es kommt nicht auf die konkreten Sprachkenntnisse an, die sie mitbringen, und auch nicht darauf, ob Kommunikationsschwierigkeiten vorliegen, sondern es geht in erster Linie um die Erwartungen der aufnehmenden Gesellschaft an die Aussiedler, perfektes Hochdeutsch bzw. aktuelles, in Deutschland übliches Regionaldeutsch zu sprechen. So äußert Dworschek (ebd., S. 23) Mitleid mit den Aussiedlern, wie mühselig es sei, wenn man „holpert“ in einer Sprache, „die man nach allem Herkommen geradezu pflichtgemäß beherrschen müsste“. Dass dieses Hochdeutsch bzw. typischer binnendeutscher „Idealdialekt“ angesichts der soziolinguistischen Situation im Herkunftsland gar nicht möglich war und daher auch von den Zuwanderern bei der Re-Migration nicht erwartet werden kann, wird nicht nur in der Bevölkerung, in den Medien, sondern auch in der Politik nicht als wichtiger Integrationsbzw. Nichtintegrationsfaktor zur Kenntnis genommen. Aus soziolinguistischer Sicht war jedoch die Nicht-Wahrnehmung ihrer realistischen Situation vor der Migration und überhöhte Erwartungen das eigentliche Sprachproblem der russlanddeutschen Aussiedler nach der Einwanderung in Deutschland.

\subsection{Spezifische Probleme der nichtdeutschstämmigen Aussiedler}

Eine andersgeartete Integrationsproblematik ist für Zuwanderer aus der Sowjetunion mit der Erst- bzw. Muttersprache Russisch typisch. Dabei handelt es sich vor allem um Familienmitglieder bzw. Familienangehörige der deutschstämmigen Russlanddeutschen, die ebenfalls den Aussiedlerstatus erhalten. (Oben wurde schon auf die identitätsbezogenen

\footnotetext{
Beleg aus dem Projektkorpus.
} 
Unterschiede zwischen den deutschstämmigen Russlanddeutschen und nichtdeutschstämmigen Zuwanderern aus Russland hingewiesen, vgl. 2.3). Zu der Gruppe, die nicht deutschstämmig ist, aber als Aussiedler aus der ehemaligen Sowjetunion in Deutschland lebt, gehören sowohl ethnische Russen als auch Kasachen, Kirgisen, Tataren und Zuwanderer anderer Nationalitäten aus der ehemaligen Sowjetunion. Es existieren keine Zahlen, die eine Vorstellung über die Größe dieser Gruppe geben würden, es kann jedoch davon ausgegangen werden, dass sie relativ groß ist, da die Zahl der deutsch-russischen Mischehen in den letzten Jahrzehnten rapide angestiegen ist. Hinsichtlich der sprachlichen Probleme der Einwanderungsgeneration gehören diese Aussiedler der Gruppe zwei an (vgl. oben): Sie sprachen bei der Einwanderung keinen russlanddeutschen Dialekt, sondern etwas Hochdeutsch und vor allem Russisch als Staatssprache des Herkunftslands. Je nach ethnischer Abstammung beherrschten sie in manchen Fällen auch die entsprechende Landessprache einer der Sowjetrepubliken.

Die russischsprachigen Zuwanderer dieser Gruppe der Aussiedler haben das klassische „Sprachproblem“ der Einwanderungsgeneration: mangelnde Kenntnisse der Zielsprache Deutsch. Die meisten hatten Kenntnisse des Deutschen als Fremdsprache in minimalem Ausmaß, die sie an sowjetischen Schulen im Rahmen des Deutschunterrichts erworben haben. In Deutschland wird der Spracherwerb des Deutschen fortgesetzt, zumeist ungesteuert. Trotz der Sprachkurse für Aussiedler ist das Standarddeutsch dieser Zuwanderer durch den charakteristischen russischen Akzent, im Sinne von bestimmten Aussprachebesonderheiten und grammatischen Abweichungen, gekennzeichnet und von den Einheimischen ohne Schwierigkeit als slawisch beeinflusste Varietät wahrnehmbar. Unter diesem Gesichtspunkt ist auch diese Zuwanderung von der Stigmatisierung auf jeden Fall betroffen. Doch scheint die Hauptproblematik dieser Zuwanderer nicht primär in dieser Außenwahrnehmung zu liegen, sondern vor allem in tatsächlichen Sprachproblemen im Sinne von Sprachdefiziten und die dadurch entstehenden Kommunikationsprobleme. Für die meisten ist (auch) dadurch der Zugang zu besseren Berufen erschwert.

Angesichts der Größe der russischsprachigen Gemeinschaft und des relativ breiten Angebots an russischsprachiger Infrastruktur können sich die Zuwanderer dieser Gruppe allerdings in ihre Migrationsnetzwerke zurückziehen. Anders als bei den deutschstämmigen Russlanddeutschen nehmen diese Migranten die Möglichkeit des Rückzugs als positive Möglichkeit der persönlichen Verwirklichung ohne Identitätsunsicherheiten wahr. Dieser Teil der russischsprachigen Migrantenpopulation in Deutschland gehört also zu den Einwanderungsgruppen, die zum Aufbau von Selbstwertgefühlen und Identitätssicherheit eine Alternative zur Aufnahmegesellschaft haben, im Gegenteil zu Russlanddeutschen deutscher Herkunft.

\subsection{Deutsch-russische Mischfamilien mit russischer „Nationalität“}

Von den ethnisch-russischen Zuwanderern zu unterscheiden sind Aussiedler, die aus Mischfamilien stammen und die aus migrationslinguistischer Sicht ebenfalls zur Gruppe 2 (vgl. oben) gehören. Bei dieser Untergruppe handelt es sich um die deutsch-russischen Zuwanderer, bei denen ein Elternteil deutsch und der andere russisch ist. Betrachtet man die Integrationsprobleme dieser Zuwanderer in Deutschland, so sind zwei Untergruppen $\mathrm{zu}$ unterscheiden: die Zuwanderer der mittleren und älteren Generation einerseits und deren Kinder andererseits. Viele erwachsene Aussiedler der mittleren und älteren Generation stammten aus Mischfamilien, d.h. sie hatten bereits im Herkunftsland die Nationalität 
und Sprache der russischsprachigen Vorfahren der Familie angenommen. Besonders in Städten und russischen Siedlungen mit größerem deutschsprachigem Anteil gab es zahlreiche Menschen deutscher Abstammung mit russischer Nationalität - und z.T. deutschrussischer Identität mit russischem Anteil. Diese Zahl hat sich in der darauffolgenden Generation noch vergrößert, und so kam es dazu, dass die Vorfahren der ab 1990 nach Deutschland eingewanderten Kinder von Menschen mit Aussiedlerstatus bereits in der Großeltern- und Elterngeneration nicht deutscher Abstammung sind. Das erklärt zumindest teilweise, warum ein Teil von zugewanderten jungen Aussiedlern sich für die Pflege und Förderung der russischen Sprache und Kultur einsetzt, was bei der einheimischen deutschen Bevölkerung nicht immer auf Verständnis stößt, insbesondere wenn es sich um deutliche Anzeichen für den russischen Sprachgebrauch und russischen Akzent handelt (Eichinger et al. 2009).

Leider wird die Heterogenität der Einwanderungsgeneration in Bezug auf den ethnischen Hintergrund gerade in Untersuchungen aus sprachlich-soziolinguistischer Sicht häufig außer Acht gelassen. In den letzten 10 bis 15 Jahren lässt sich dieser Trend beobachten. Das führt dazu, dass die eigentliche Aussiedlerproblematik, die auf die ethnische Identität als Deutsche zurückgeht, nicht als relevanter Faktor gesehen wird und es meistens nur darum geht, die „Russisch-Sprachigkeit“ der Russlanddeutschen zu untersuchen. Außerdem werden die sehr unterschiedlichen ethnischen Gruppen der Russischsprachigen häufig undifferenziert in empirische Sprachforschungen einbezogen, was wiederum dazu führt, dass der Faktor der ,sociolinguistic continuity from old to new homeland“ (Clyne 2007) nicht ausreichend berücksichtigt wird.

\section{Zu diesem Band}

Der vorliegende Band umfasst die Beiträge der interdisziplinären Arbeitstagung „Sprache - Identität - Integration“, die am 14. und 15. November 2013 im Institut für Deutsche Sprache stattgefunden hat. ${ }^{8}$

Die Tagung setzte sich das Ziel, vom interdisziplinären Gesichtspunkt aus einen Beitrag zur Klärung der Spezifik der Aussiedlermigration in der Gegenwart zu leisten. Denn obwohl die „russlanddeutschen Aussiedler“ im Rahmen der russischsprachigen Zuwanderungspopulation die größte Gruppe darstellen, wird ihnen in der aktuellen Forschung bisher relativ wenig Aufmerksamkeit geschenkt. Es liegen zwar einige Forschungsergebnisse in den Bereichen Sprachwissenschaft/Dialektologie/Kontaktlinguistik und in der Soziologie vor, doch die aktuelle Forschungslandschaft stellt sich - gemessen an der Größe der Zuwanderungsgruppe der Aussiedler - mehr als bescheiden dar. Insbesondere die interne Differenzierung bleibt mehr oder weniger unerwähnt bzw. nicht berücksichtigt. Die spezifische Migrationskonstellation dieser Zuwanderer erscheint jedoch für ihre Integration als besonders relevant, wie in den vorherigen Abschnitten dieser Einführung ausgeführt wurde. Denn einerseits handelt es sich um „Deutschstämmige“, die mit einer spezifischen Einstellung und mit zum Teil spezifischen Deutschkenntnissen nach Deutschland einwandern (,koethnische Remigranten“), bei denen somit bestimmte Vorteile für die Integration zu erwarten wären. Andererseits weisen diese Zuwanderer in bestimmten Zusammenhängen Merkmale der regulären Migration auf, d.h. dass auch bei Aussiedlern typische, migrationsbedingte Probleme wie z.B. mangelnde Integration und fehlende Akzeptanz von

8 Mit Ausnahme des Beitrags von Katharina Dück. 
Seiten der aufnehmenden Gesellschaft auftreten. Ein wichtiger Diskussionspunkt der Tagung waren daher auch die verschiedenen internen Differenzen in Bezug auf die verschiedenen Zuwanderungsgruppen der russlanddeutschen Aussiedler, wie sie oben (Abschnitt 2 und 3) dargestellt wurden.

Die vorliegenden Beiträge behandeln verschiedene Aspekte der soziolinguistischen Situation und des Spracherwerbs in Bezug auf die oben besprochenen Untergruppen der russlanddeutschen Zuwanderungspopulation in Deutschland. Im Beitrag von Alexander Prediger geht es um den Erwerb von regionalen Varietäten des Deutschen bei der großen Gruppe der russischsprachigen Zuwanderer in Süddeutschland. Sie sind dem Status nach zwar Aussiedler, hatten bis zur Migration jedoch keinerlei oder kaum Kontakte zur deutschen Sprache und verfügten folglich auch nicht über muttersprachliche Kenntnisse von irgendwelchen russlanddeutschen Dialekten. Es handelt sich hier im Sinne der oben geschilderten Darstellung um die nichtdeutschstämmigen Aussiedler der Einwanderungsgeneration. Eine gezielte Auswahl von Probanden (wie Familienangehörige von Aussiedlern und Spätaussiedlern), die aus sprachbiografischen Gründen keine Kontakte zu Dialekten hatten, erlaubt empirisch fundierte Untersuchungsergebnisse zu dieser speziellen Untergruppe der Aussiedler und realistische Interpretationen in Bezug auf die Untersuchungsfrage im Vergleich zur Gruppe der deutschstämmigen Aussiedler, die mit guten Dialektkenntnissen einwandern und die in diesem Band von Nina Berend untersucht werden. Ihr Beitrag beschäftigt sich aus soziolinguistischer Perspektive mit „Re-Migranten“ - also Aussiedlern aus deutschen Sprachinseln, die bei der Einwanderung deutsche Dialekte russlanddeutscher Herkunft sprachen (,deutschstämmige Aussiedler mit Dialektkenntnissen“"). Im Mittelpunkt des Beitrags von Nataliya Soultanian und Lukas Nock stehen junge russlanddeutsche Eltern, die selbst als Kinder nach Deutschland eingewandert sind und sich - als Eltern - für die Pflege und Förderung der russischen Sprache und Kultur bei ihren Kindern einsetzen. Hier handelt es sich um russlanddeutsche Aussiedler, die vermutlich bereits in der zweiten bzw. dritten Generation in der Familie russische Vorfahren haben. Auch im Beitrag von Katharina Dück steht die zweite Generation im Mittelpunkt. In diesem Beitrag geht es um den Zusammenhang zwischen der Sprache und ethnischer Identität der z.T. als Kinder nach Deutschland eingewanderten jungen russlanddeutschen Aussiedler.

Abgerundet wurde die Tagung durch das Programm „Deutsch... aber nicht ganz“, in dem die Kabarettistin Lilia Tetslau, selbst zugewanderte Russlanddeutsche, durch das „Labyrinth der Integration“" der Russlanddeutschen führt (vgl. Abb. 1). ${ }^{9}$ 9 Vgl. www.tetslau-joey.de/kabarett/index.html. In Leontiy (2013) liegt die Analyse einer Episode (Video-
Aufnahme) aus diesem Programm vor. 


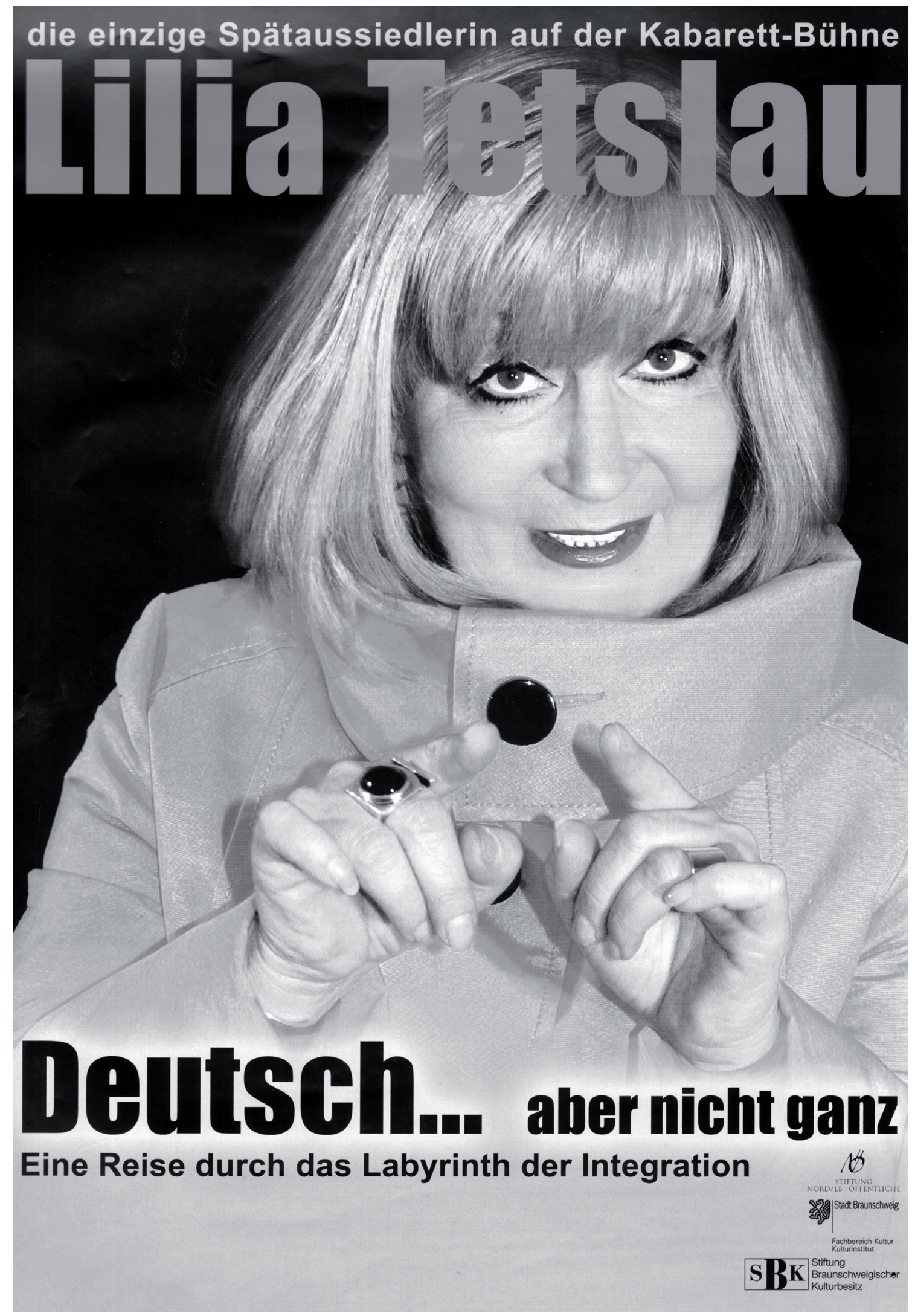

Abb. 1: Plakat zum Bühnenprogramm der Kabarettistin Lilia Tetslau 
Nur auf dem Hintergrund der Vor-Migrations-Konstellation der russlanddeutschen Minderheit in der ehemaligen Sowjetunion, ihrer spezifischen Zweisprachigkeitssituation und der Gestaltung der deutsch-russischen Sprachkontakte ist eine adäquate Interpretation des Sprachverhaltens und der Integrationschancen dieser Zuwanderungspopulation möglich. Gerade angesichts der unterschiedlichen Spracherfahrungen der Vorfahren im Herkunftsland können nach der Migration unterschiedliche Einstellungen vorherrschen. Die Integrationsdifferenzen können solche relevanten Aspekte betreffen wie den Erwerb der Zielsprache und deren regionalen Varietäten, den Gebrauch, Erhalt und Förderung der Herkunftssprache Russisch sowie die Art und Weise der deutsch-russischen Sprachmischungen. Eine adäquate Beschreibung erfordert die Berücksichtigung der internen Differenzierung der Aussiedler-Zuwanderung, auch wenn sich keine scharfen Grenzen zwischen den einzelnen Untergruppen ziehen lassen.

\section{Literatur}

Bausinger, Hermann (1956): Beharrung und Einfügung. Zur Typik des Einlebens der Flüchtlinge. In: Jahrbuch für Volkskunde der Heimatvertriebenen 2, S. 9-16.

Berend, Nina (1977): Interferirujuščee vlijanie nemeckich govorov na russkie rečevye proizvedenija ich nositelej. In: Jedig, Hugo (Hg.): Voprosy struktury germanskich jazykov 2. Omsk. S. 9-17.

Berend, Nina (1998): Sprachliche Anpassung. Eine soziolinguistisch-dialektologische Untersuchung zum Russlanddeutschen. Tübingen: Narr. (= Studien zur deutschen Sprache 14).

Berend, Nina (2012): Migration und Dialektwandel. Aspekte der jüngsten Sprachgeschichte des Russlanddeutschen. In: Bär, Jochen A./Müller, Marcus (Hg.): Geschichte der Sprache - Sprache der Geschichte. Probleme und Perspektiven der historischen Sprachwissenschaft des Deutschen. Oskar Reichmann zum 75. Geburtstag. Berlin: Akademie. S. 609-628. (= Lingua Historica Germanica 3).

Clyne, Michael (2007): Sociolinguistic Continuity from Old to New Homeland: Factors in Language Maintenance and Shift Seen from the Australian Situation. In: Darquennes, Jeroen (Hg.): Contact Linguistics and Language Minorities. St. Augustin: Asgard. S. 91-102. (= Plurilingua XXX).

Currle, Edda (2006): Theorieansätze zur Erklärung von Rückkehr und Remigration. In: soFid. Migration und ethnische Minderheiten 2006/2, S. 7-22.

Cyrus, Norbert et al. (2005): Opportunity Structures for Immigrants' Active Civic Participation in the European Union: Sharing Comparative Observations. University of Oldenburg. POLITIS-Working paper No. 2. Online: www.politis-europe.uni-oldenburg.de/download/WP2_POLITIS_CyrusGropasKosicVogel_ 2005. pdf (15.10.2012).

Diener, Irina (2003): Muttersprache - Lernerfahrungen einer russlanddeutschen Mutter und Lehrerin in Deutschland. In: Reitmeier, Ulrich (Hg.): Sprachliche Integration von Aussiedlern im internationalen Vergleich. Mannheim: Institut für Deutsche Sprache. S. 269-276.

Dworschek, Manfred (1991): Mir sagen haite „Tschuiss!“ Von verschollenen Dialekten: Die tausend rußlanddeutschen Pfingstler in Bremen kämpfen mit eigenartigen Sprachproblemen. In: taz.die tageszeitung, 25.11.1991.

Eichinger, Ludwig M. et al. (2009): Aktuelle Spracheinstellungen in Deutschland. Erste Ergebnisse einer bundesweiten Repräsentativumfrage. Mannheim: Institut für Deutsche Sprache.

Ipsen-Peitzmeier, Sabine/Kaiser, Markus (Hg.) (2006): Zuhause fremd. Russlanddeutsche zwischen Russland und Deutschland. Bielefeld: transcipt.

Hermann, Michael C./Öhlschläger, Rainer (Hg.) (2013): Hier die deutschen - dort die Russen. Integrationsprobleme russlanddeutscher Jugendlicher 250 Jahre nach dem Einladungsmanifest von Katharina ii. Baden-Baden: Nomos. 


\section{Nina Berend}

Leontiy, Halina (2013): Eine Reise durch ein Integrationslabyrinth - Potentiale des Ethnokabaretts bei der kulturellen Wissensvermittlung am Beispiel der Spätaussiedler in Deutschland. In: Weber, Tilo/Ballod, Matthias (Hg.): Autarke Kommunikation. Wissenstransfer in Zeiten von Fundamentalismen. Frankfurt/ Main: Lang. S. 191-222. (= Transferwissenschaften 9).

Strobl, Rainer (2006): Chancen und Probleme der Integration junger Aussiedler aus der früheren Sowjetunion. In: Ipsen-Peitzmeier, Sabine/Kaiser, Markus (Hg.), S. 87-109.

Woellert, Franziska et al. (2009): Ungenutzte Potenziale. Zur Lage der Integration in Deutschland. Online: www.berlin-institut.org/fileadmin/user_upload/Zuwanderung/Integration_RZ_online.pdf.

Prof. Dr. Nina Berend

Institut für Deutsche Sprache

R 5, 6-13

D-68161 Mannheim

E-Mail: berend@ids-manheim.de 